pulmonary disease. The alterations are unlikely to be secondary to the pancreatic insufficiency found in the great majority of patients with $\mathrm{CF}$ since the three patients without pancreatic insufficiency also exhibited increased $\alpha$-D-glucosidase activity levels and altered isoelectric profiles.

The results of the present investigation emphasize the importance of including appropriate pathological controls in addition to normal controls when doing biochemical studies on CF. Since appropriate pathological controls are often not included in studies on CF, it is likely that many of the biochemical abnormalities found in tissues derived from patients with $C F$ are not specific for $\mathrm{CF}$ but are secondary to chronic disease.

Acknowledgment. Deborah Christie, R.N., organized and participated in the collection of patient blood samples.

\section{REFERENCES}

1. Alhadeff JA 1980 Glycoprotein metabolism in cystic fibrosis. In: Sturgess JM (ed) Perspectives in Cystic Fibrosis. Proceedings of the Eighth International Congress on Cystic Fibrosis. Imperial Press, Ontario, pp 35-43
2. Alhadeff JA, Thom D, Holzinger RT 1981 Activity levels and properties of acid $\alpha$-glucosidase from liver and neutral $\alpha$-glucosidase from sera of cystic fibrosis patients and controls. Clin Chim Acta 117:227-237

3. Antonowicz I, Sippell WG, Shwachman H 1972 Cystic fibrosis: lysosomal and mitochondial enzyme activities of lymphoid cell lines. Pediatr Res 6:803812

4. Casola L, DiMatteo G, Romano M, Rutigliano B, Mastella G 1979 Glycosidases in serum of cystic fibrosis patients. Clin Chim Acta 94:83-88

5. DiMatteo G, Romano M, Mastella G, Castellani E, Rutigliano B, Casola L 1979 Chemical comparison of normal and cystic fibrosis meconium: quantitative and qualitative analysis of carbohydrate splitting enzymes. Monogr Pediatr 10:19-26

6. Hosli P, Vogt E 1977 Cystic fibrosis: leakage of lysosomal enzymes and of alkaline phosphatase into the extracellular space. Biochem Biophys Res Commun 79:741-748

7. Riordan JR, Alon N, Duthie M, Maler T, Buchwald M 1980 Hydrolytic enzymes as markers for cystic fibrosis. In: Sturgess JM (ed) Perspectives in Cystic Fibrosis. Proceedings of the Eighth International Congress on Cystic Fibrosis. Imperial Press, Ontario, pp 313-321

8. Shwachman H, Kulczycki LL 1958 Long-term study of one hundred five patients with cystic fibrosis. Am J Dis Child 96:6-15

9. Talamo RC, Rosenstein BJ, Berninger RW 1983 Cystic fibrosis. In Stanbury JB, Wyngaarden JB, Frederickson DS, Goldstein JL, Brown MS (eds) The Metabolic Basis of Inherited Disease, 5th ed. McGraw-Hill, New York, pp 1889-1917

\title{
Day-to-Day Pneumogram Variability
}

\author{
CARL E. HUNT, ROBERT T. BROUILLETTE, KIANG LIU, AND LINDA KLEMKA \\ Departments of Pediatrics, Community Health and Preventative Medicine, Northwestern University Medical \\ School and Children's Memorial Hospital, Chicago, Illinois 60614
}

\begin{abstract}
To determine day-to-day variability in respiratory pattern, we obtained 188 comparisons of pneumograms performed during two successive 24-h intervals. The respiratory pattern values calculated were total duration of brief apnea (apnea density), periodic breathing episodes, longest apnea, number of apneas $>11 \mathrm{~s}$, and number of apneas $>15 \mathrm{~s}$. For day 1 values for apnea density, periodic breathing, and longest apnea which were within the 90th percentile for normal infants at age 1 month, day 2 values fell outside that range in $4.3,3.5$, and $18 \%$ of comparisons, respectively. There was a systematic tendency for the day 2 values to be less than the day 1 values. For each parameter, the day 2 range was lower, the slope of the day 2 versus day 1 regression line was significantly less than $1(p<0.01)$ and the $y$-intercept was significantly greater than zero $(p<0.01)$. Knowledge of day-to-day pneumogram variability should be helpful in interpreting individual pneumogram results and in assessing the clinical usefulness of pneumogram recordings. (Pediatr Res 19: 174-177, 1985)
\end{abstract}

Received November 7, 1983; accepted September 27, 1984.

Address for reprints Carl E Hunt, M.D., Division of Neonatology, Children's Memorial Hospital, 2300 Children's Plaza, Chicago, IL 60614

Supported by the Children's Research Guild and the Lillian S. Wells Foundation.

\author{
Abbreviations \\ SIDS, sudden infant death syndrome \\ AOI, apnea of infancy \\ $\mathbf{A}_{6} / \mathbf{D} \%$, apnea density
}

Two-channel recordings of ECG and thoracic impedence (pneumograms) are commonly performed in infants with symptomatic apnea and in asymptomatic infants considered to be at increased risk for SIDS. Based primarily on single 24-h recordings, Kelly and coworkers $(3,4)$ documented significantly greater periodic breathing in AOI infants and in subsequent siblings compared to control infants. In earlier reports, we confirmed the presence of pneumogram abnormalities in AOI compared to control infants (2), and documented improvement in these abnormalities with theophylline (1).

Investigators have used various methods for printing and displaying pneumogram recordings and different criteria for pneumogram interpretation. In addition to uncertainty as to the upper normal limit for the various calculated parameters, there are no reported studies of day-to-day variability in pneumogram results. Although two previous pneumogram studies in normal infants $(2,5)$ did include some serial recordings, variability could 
not be assessed because the time interval between recordings was too long to attribute any observed differences only to individual variability. We now report the results of 188 comparisons of pneumogram recordings performed on successive days in 164 infants encompassing a wide range of possible respiratory patterns.

\section{METHODS}

Two consecutive 24-h recordings were performed in 164 infants, 96 boys and 68 girls. Repeat recordings were obtained in 21 infants, resulting in a total of 188 comparisons. Among the 164 infants, 109 were AOI patients, 30 were appropriate for gestational age preterm infants (birth weight $<1500 \mathrm{~g}$ ) evaluated approximately 1 wk prior to neonatal ICU discharge, 22 were asymptomatic siblings of prior SIDS victims, and three were normal infants. Initial pneumograms in the AOI group were performed in the hospital at a mean age of $7.7 \pm 7.1$ (SD) wk. Initial pneumograms in the asymptomatic siblings and normal infants were performed at home, at a mean age of $6.4 \pm 8.8 \mathrm{wk}$. The 24 repeat pneumograms in 21 infants all were performed at home.

The inpatient and home pneumograms were performed under normal ambient conditions for that location. According to the standard routine for all pneumogram recordings, intermittent behavioral state observations were made by the nurse or parent caretaker. Sleep time was determined from a log of sleep intervals as recorded by the caretaker. Within each $24-\mathrm{h}$ recording, all quiet baseline intervals occurring during the periods indicated as sleep time by the log were analyzed for five respiratory parameters: brief apnea, periodic breathing episodes, longest apnea, number of apneas $>11 \mathrm{~s}$, and number of apneas $>15 \mathrm{~s}$. Brief apneas were quantitated as $\mathrm{A}_{6} / \mathrm{D} \%$, the total duration of all $\geq 6 \mathrm{~s}$ respiratory pauses expressed as a percent of total sleep time $(1,6,7)$. Periodic breathing was calculated as the number of episodes/100 min (1). At least $8 \mathrm{~h}$ of sleep time were available for analysis in each pneumogram.

For each infant, each 24 -h recording was analyzed separately and the day 1 results for each of the five parameters were compared to the day 2 results by paired $t$ test. For each parameter, the day 1-day 2 group results also were compared by standard regression analysis. To permit display of the full range of values obtained for $\mathrm{A}_{6} / \mathrm{D} \%$, periodic breathing and longest apnea, the actual day 1-day 2 comparisons were plotted on a log scale. Group results are expressed as the mean \pm SD.

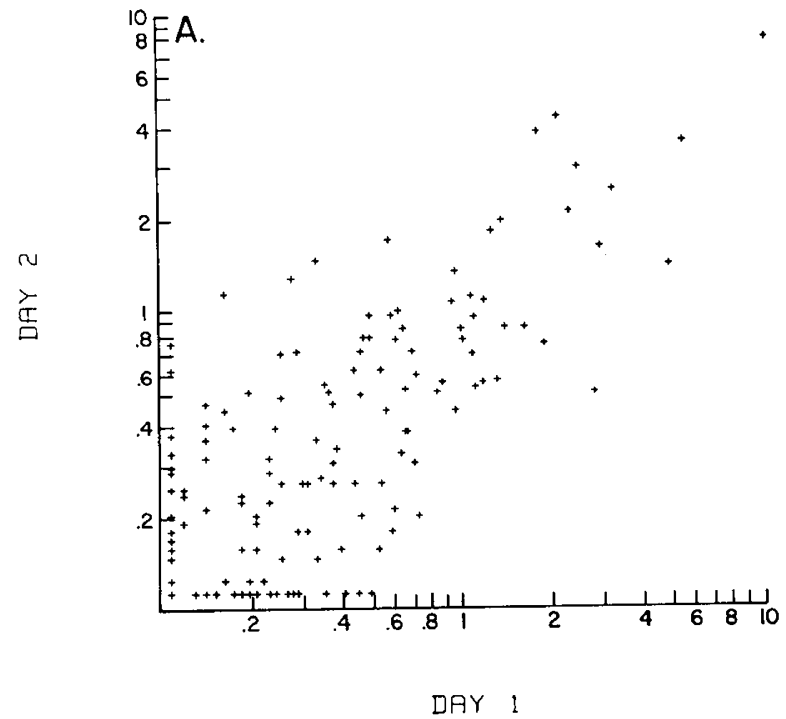

\section{RESULTS}

Total sleep time was $12.2 \pm 2.9 \mathrm{~h}$ for day 1 and $12.15 \pm 3.0 \mathrm{~h}$ for day 2. The range for $\mathrm{A}_{6} / \mathrm{D} \%$ was $0-8.9 \%$ on day 1 and 0 $6.7 \%$ on day 2 . The range for periodic breathing episodes $/ 100$ min was $0-10.4$ on day 1 and $0-9.2$ on day 2 . The range for longest apnea was 5-51 s on day 1 and 5-34 s on day 2. The range for number of apneas $>11 \mathrm{~s}$ was $0-38$ on day 1 and $0-17$ on day 2. The range for number of $>15 \mathrm{~s}$ apneas was $0-18$ on day 1 and $0-8$ on day 2 . The mean group differences between day 1 and day 2 were not significant by paired $t$ test for any of the five breathing pattern parameters calculated.

To assess directly the extent to which results of a single 24-h recording are representative, we analyzed the frequency with which the day 2 results remained within the same range as on day 1 (Table 1). For this purpose we used the 90th and 95th percentiles for the values observed in normal infants (2) at 1 month of age. Depending on the maximum day $1 \mathrm{~A}_{6} / \mathrm{D} \%$ value selected, $95.7-96.4 \%$ of the day 2 results were within the same range. For periodic breathing, $96.5-98.8 \%$ of day 2 values were within the day 1 range. For longest apnea, $82-88 \%$ of day 2 values were within the day 1 range.

The day 1 and day 2 values for $\mathrm{A}_{6} / \mathrm{D} \%$, periodic breathing, and longest apnea were also compared using standard regression

Table 1. Direct assessment of day-to-day variability according to the frequency with which day 2 results remain within the same range as day $1^{*}$

\begin{tabular}{lccc}
\hline \multicolumn{1}{c}{ Parameter } & $n$ & Day 1 & $\begin{array}{c}\text { Day } 2 \\
\text { \% in same range }\end{array}$ \\
\hline $\mathrm{A}_{6} / \mathrm{D} \%$ & 161 & $<0.79$ & 95.7 \\
& 166 & $<0.92$ & 96.4 \\
Periodic breathing & 170 & $<2.4$ & 96.5 \\
(episodes 100/min) & 173 & $<2.9$ & 98.8 \\
Longest & 136 & $<11$ & 81.6 \\
apnea (s) & 152 & $<12$ & 88.2 \\
No. apneas & 146 & 0 & 88.4 \\
$>11$ & 163 & 1 & 93.9 \\
No. apneas & 171 & 0 & 96.5 \\
$>15 \mathrm{~s}$ & 171 & 0 & 96.5 \\
\hline
\end{tabular}

* For each parameter, the two day 1 maximum limits selected represent the 90th and 95th percentiles for normal infants at 1 month of age (2).

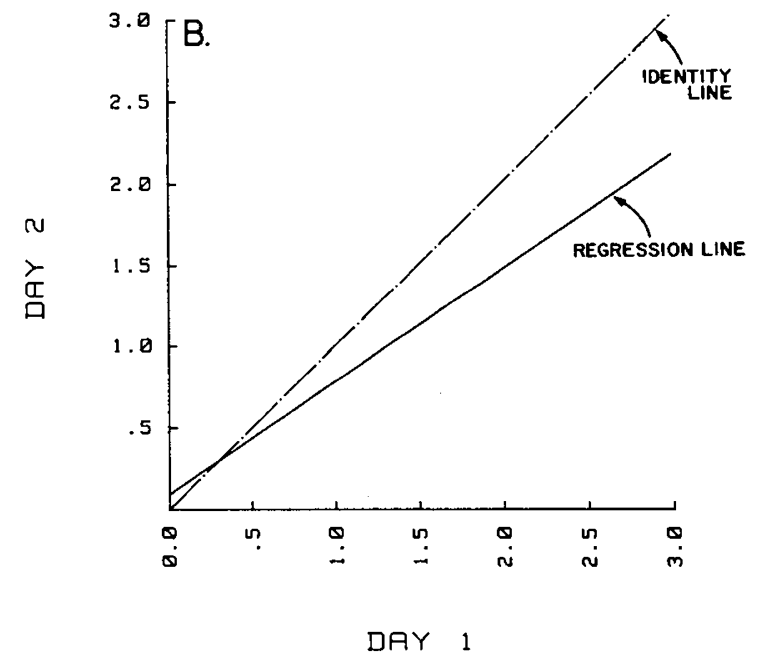

Fig. 1. Comparison of day 1 and day 2 values for $\mathrm{A}_{6} / \mathrm{D} \%$. $A$, individual data points for the 188 comparisons. For this log scale, all values $<0.1 \%$ are plotted as $0.1 \%$. Identical points are superimposed. $B$, calculation of theoretical variability. The slope of the regression line is significantly less than 1 (identity), $p<0.01 . \mathrm{Y}=0.69 \times+0.09, \mathrm{r}=0.85$. 
analysis. For all three parameters, the group values on day 2 were consistently less than on day 1 . The slope of the regression line for $A_{6} / D \%$ (Fig. 1) was significantly greater than zero and significantly less than $1(p<0.01)$ and the y intercept $(0.09 \%)$ was significantly greater than zero, indicating that the trend for day 2 results was significantly less than day 1 . The slope of the regression line for periodic breathing was significantly greater than zero and significantly less than $1(p<0.01)$ and the $y$ intercept $(0.15)$ was significantly greater than zero, indicating that the trend for day 2 results was also significantly less than day 1 (Fig. 2). Similar to the results for $A_{6} / D \%$ and periodic breathing, the slope of the regression line for longest apnea (Fig. 3 ) was also significantly greater than zero and significantly less than one $(p<0.01)$ and the $\mathrm{y}$ intercept $(3.8 \mathrm{~s})$ was significantly greater than zero $(p<0.01)$, indicating that the trend for day 2 results was significantly less than day 1 . The slopes of the regression lines for number of apneas $>11 \mathrm{~s}$ and for number of apneas $>15 \mathrm{~s}$ were significantly greater than zero and significantly less than $1(p<0.01)$, again indicating that the trend for day 2 results was significantly less than day 1 .

Additional statistical analyses were performed to determine whether regression toward the mean might explain why the slopes were less than 1 (identity). We repeated the regression analyses for $\mathrm{A}_{6} / \mathrm{D} \%$, periodic breathing, and longest apnea after excluding all values $\geq 0.5 \%, \geq 1.5$ episodes $/ 100 \mathrm{~min}$, and $>11 \mathrm{~s}$, respectively. The results (Table 2) were similar; the slopes remained significantly less than $1(p<0.01)$.

\section{DISCUSSION}

One of the major questions related to the pneumogram is its reliability. Our study addresses this issue by evaluating day-to-
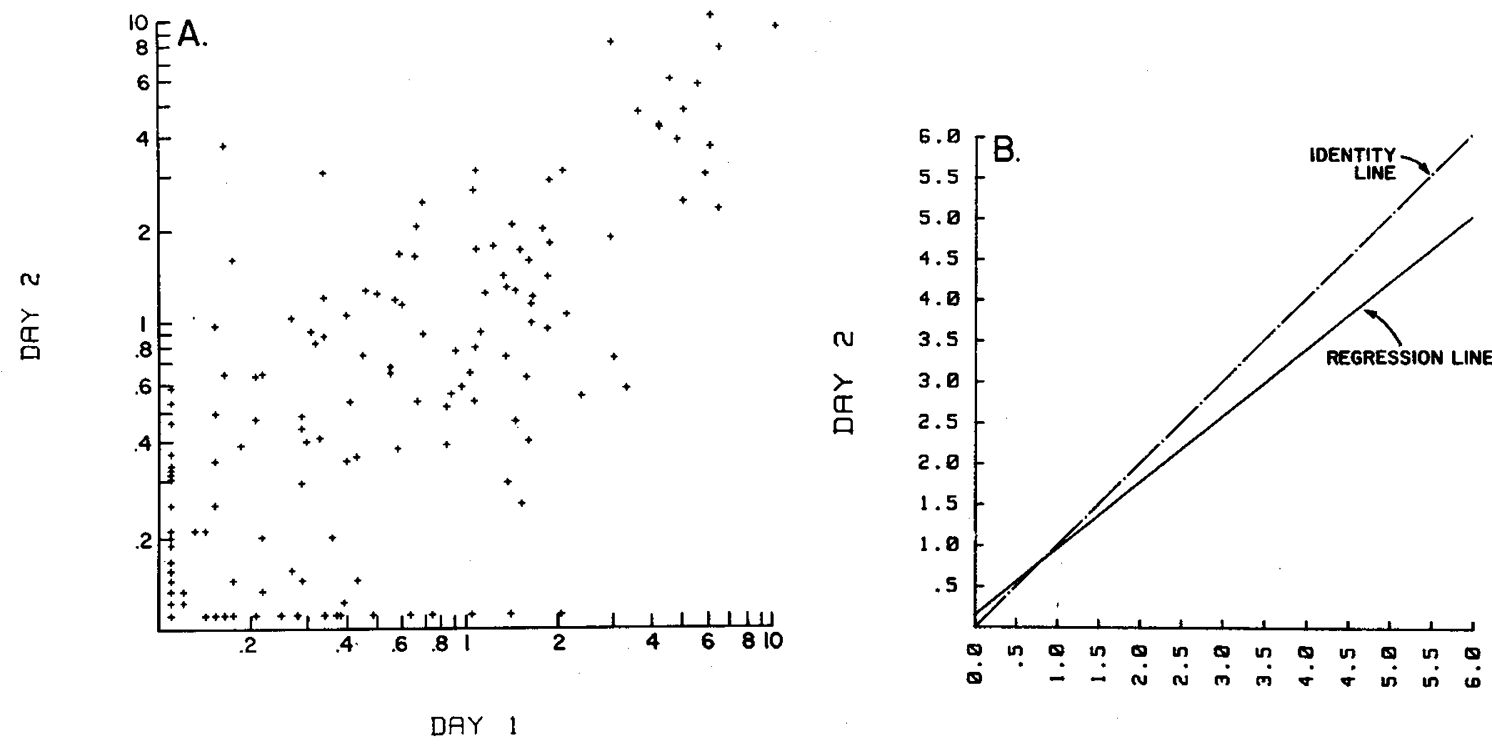

DAY

Fig. 2. Comparison of day 1 and day 2 values for periodic breathing episodes/100 min. $A$, individual data points for all 188 comparisons. All values $<0.1$ are plotted as 0.1 . Identical points are superimposed. $B$, calculation of theoretical variability. The slope of the regression line is significantly less than 1 (identity), $p<0.01 . Y=0.81 \times+.15, r=0.82$.
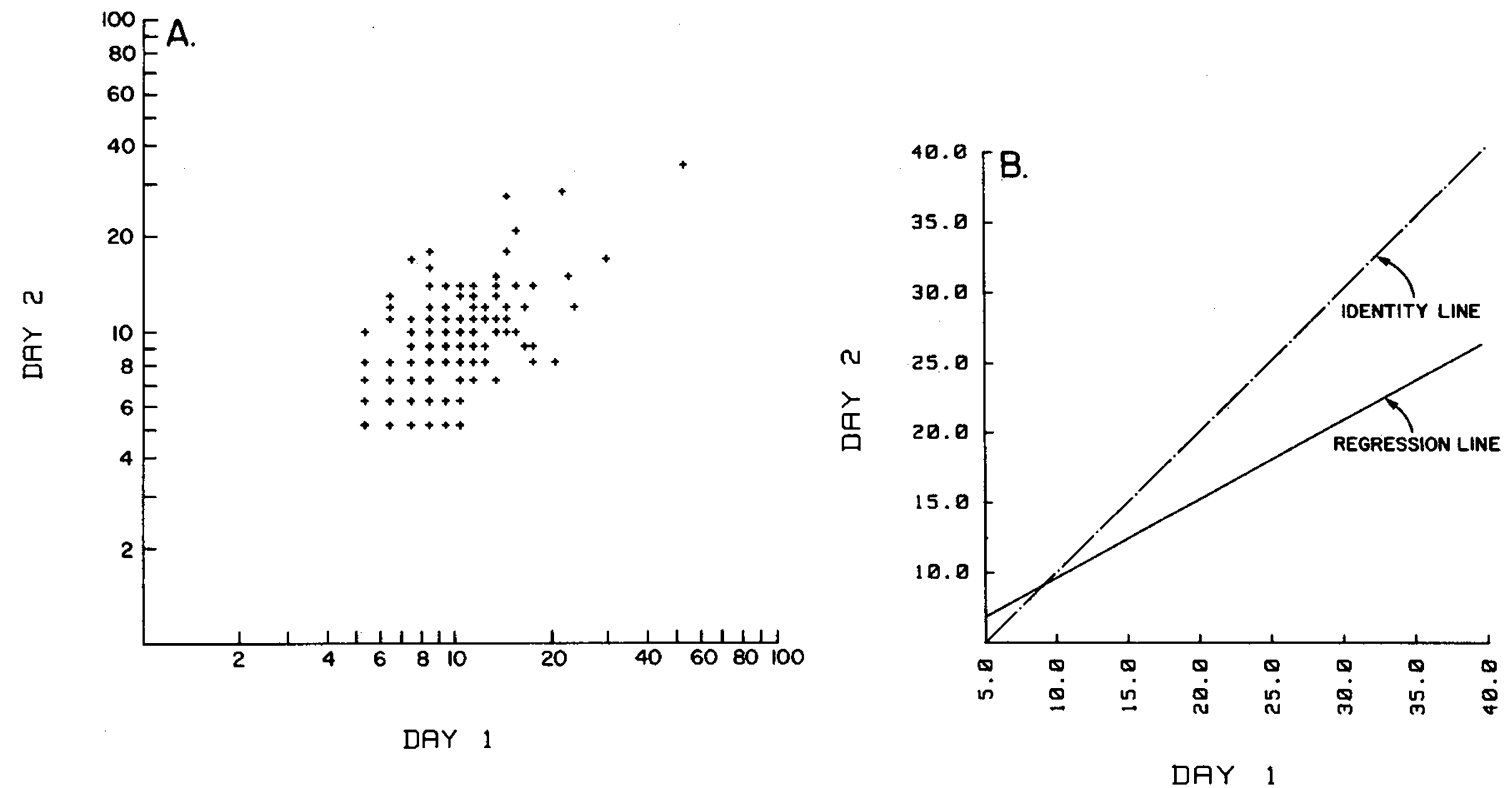

Fig. 3. Comparison of day 1 and day 2 values for longest apnea (s). $A$, individual data points for 187 comparisons. Identical values are superimposed. $B$, calculation of theoretical variability. The slope of the regression line is significantly less than 1 (identity), $p<0.01$. $\mathrm{Y}=0.56 \times+$ $4.0, \mathrm{r}=0.65$. 
Table 2. Slopes for $A_{6} / D \%$, periodic breathing (episodes $/ 100$ min) and longest apnea*

\begin{tabular}{lcc}
\hline \multicolumn{1}{c}{ Parameter } & $n$ & Slope $\dagger$ \\
\hline $\mathrm{A}_{6} / \mathrm{D} \%$ & 188 & $0.691 \pm 0.03$ \\
$\quad$ All data & 129 & $0.407 \pm 0.08$ \\
$\quad$ Both days $<0.5$ & & \\
Periodic breathing & 188 & $0.807 \pm 0.04$ \\
$\quad$ All data & 146 & $0.470 \pm 0.07$ \\
$\quad$ Both days $<1.5$ & 187 & $0.559 \pm 0.05$ \\
Longest apnea & 134 & $0.464 \pm 0.08$ \\
$\quad$ All data & \\
$\quad$ Both days $<11 \mathrm{~s}$ &
\end{tabular}

* The slopes remain significantly less than 1 (identity) even when the extreme values are excluded, indicating that the lower day 2 values cannot be explained by "regression toward the mean."

$\dagger \pm \mathrm{SE}$ of regression coefficient.

day variability in respiratory pattern in 164 infants. We are not aware of any previous published studies of day-to-day pneumogram variability. By direct analysis, day 1 results for $A_{6} / D \%$, periodic breathing, and longest apnea that were within the 95 th percentile for normal infants (2) remained in the same range in $90 \%$ or more of the comparisons obtained on day 2 .

There is a systematic trend toward lower day 2 values compared to day 1 . For each parameter the day 2 range is lower than day 1 , the slope of the regression line is significantly less than 1 $(p<0.01)$ and the $y$ intercept is significantly greater than zero $(p<0.01)$. Although such an adaptation phenomenon has not been previously observed for pneumograms, and was not expected, an adaptation or "first night" effect has been observed for a variety of other biologic measurements. We have no explanation to account for a "first night" effect with pneumogram recordings. Whether the relationship between clinical status, SIDS risk, and respiratory pattern should be assessed using day 1 or day 2 pneumogram values is an important question that will need to be addressed in subsequent studies. Fortunately, however, false-negative day 1 results are unlikely since only $3.6 \%$ of day $1 \mathrm{~A}_{6} / \mathrm{D} \%$ values $<0.92,1.2 \%$ of day 1 periodic breathing episodes $<2.9$ episodes $/ 100 \mathrm{~min}$, and $11.8 \%$ of longest apnea $<12$ s, will be above those limits on day 2 (Table 1 ).

It is necessary to be certain that the systematic tendency toward lower day 2 values which we have observed is a physiologic or "first night" phenomenon rather than "regression to the mean." Our results are not normally distributed; there is skewness to the right due to some extreme values. To determine whether inclusion of these extreme values could result in lower day 2 values due to the phenomenon of "regression toward the mean," we recalculated each slope after excluding all extreme values. These truncated slopes (Table 2) were even lower than the original slopes, confirming that the significantly lower day 2 values cannot be explained by "regression toward the mean."

The pneumogram parameters which we analyzed for this study are the same as in previous studies $(1,2)$. Our analyses of $\mathrm{A}_{6} /$ $\mathrm{D} \%$, periodic breathing, and longest apnea in apnea of infancy indicate significantly greater respiratory pattern abnormalities than in normal infants at either 1 or 3 months of age (2). Although this study was not designed to assess further the relationship between respiratory pattern abnormalities and potential risk for SIDS, subsequent studies of this relationship should be facilitated by improved knowledge of day-to-day pneumogram variability.

Acknowledgments. The authors thank Donna Hanson and Stephen Lee for their technical assistance and data analysis, and Susan Seidler and Judy Carbone for their assistance in preparation of this manuscript.

\section{REFERENCES}

1. Hunt CE, Brouillette RT, Hanson D 1983 Theophylline improves pneumogram abnormalities in infants at risk for SIDS. J Pediatr 103:969-74

2. Hunt CE, Brouillette RT, Hanson D, Stein IM, David RJ, Weissbluth M. Home pneumograms in normal infants versus apnea of infancy. $J$ Pediatr (in press)

3. Kelly DH, Shannon DC 1979 Periodic breathing in infants with near-miss sudden infant death syndrome. Pediatrics 63:355-360

4. Kelly DH, Walker AM, Cahen L, Shannon DC 1980 Periodic breathing in siblings of sudden infant death syndrome victims. Pediatrics 66:515-520

5. Stein IM, White A, Kennedy JL, Merisalo RL, Chernoff H, Gould JB 1979 Apnea recordings of healthy infants at 40,44 , and 52 weeks postconception. Pediatrics 63:724-730

6. Steinschneider A 1977 Prolonged sleep apnea and respiratory instability: a discriminative study. Pediatrics 59:962

7. Steinschneider A, Weinstein SL, Diamond E 1982 The sudden infant death syndrome and apnea/obstruction during neonatal sleep and feeding. Pediatrics $70: 858-863$ 